\title{
Guest editorial: Cooperative networks for stem cell homeostasis in normal and malignant hematopoiesis: from metabolism to epigenetics
}

\author{
Atsushi Hirao1 \\ Received: 5 April 2016 / Accepted: 7 April 2016 / Published online: 19 April 2016 \\ (C) The Japanese Society of Hematology 2016
}

Hematopoietic stem cells (HSCs) are defined as cells that have the ability to perpetuate undifferentiated status through self-renewal and to develop into mature cells through differentiation. Fine-tuning of self-renewal and differentiation programs, mediated by cooperative networks of intrinsic and extrinsic factors, contributes to stem cell homeostasis in vivo [1, 2]. Accumulating evidence has revealed the presence of "stemness" signals; that is, molecules and signaling pathways that play critical roles in maintaining the undifferentiated properties of HSCs. Common mechanisms may regulate stemness in HSCs and leukemias: although leukemia stem cells (LSCs) appear to be heterogeneous, their gene expression profiles closely resemble those of normal HSCs or progenitors. Understanding the commonalities and critical differences between HSCs and LSCs in the regulation of stemness is required to find the therapeutic window. In this issue, four articles review the physiological and pathophysiological roles of metabolism and epigenetic regulation in hematopoiesis. The articles include current basic knowledge and recent topics in the field.

To date, numerous extrinsic factors have been identified as critical regulators of HSC homeostasis in specific microenvironments (Fig. 1). Although detailed studies have revealed that critical cytokines/chemokines are essential for the development, survival, and self-renewal of HSCs, the regulation of these signals is not necessarily simple. For example, there is a paradox in the relationship between the cell cycle of HSCs and the cytokines, because the majority

Atsushi Hirao

ahirao@staff.kanazawa-u.ac.jp

1 Division of Molecular Genetics, Cancer Research Institute, Kanazawa University, Kanazawa, Ishikawa 920-1192, Japan of HSCs in vivo are quiescent, even though cytokines known to regulate HSCs, such as c-Kit and mpl ligand, are considered to be growth factors that stimulate cell cycle progression. How such factors maintain quiescent HSCs is still a mystery. Presumably, direct interactions of the HSCs with neighboring cells (niche cells); secreted factors such as nutrients, hormones, and metabolites; or oxygen tension modulate the signals of growth factors in the HSCs.

In the niche, extrinsic factors coordinate multiple processes that are essential for HSC homeostasis and are mediated by metabolic regulation. Several studies have suggested that HSCs utilize glycolysis, whereas oxidative phosphorylation becomes dominant in progenitors. Like HSCs, LSCs exhibit low levels of ROS; however, unlike HSCs they appear to have low glycolytic flow, indicating that there are critical differences in metabolic regulation between these cell types. In this issue, Jiang et al. argue that knowledge of the molecular mechanisms of metabolic regulation mediated by key molecules, e.g., HIF1/2, AMPK, and mTOR, will provide novel therapeutic approaches for eradiation of LSCs [3].

Multistep processes of hematopoiesis and leukemogenesis are controlled by epigenetics, which regulates gene expression by increasing or decreasing DNA methylation or post-translational modifications of histone core proteins. DNA methylation proceeds by the transfer of a methyl group onto the C5 position of the cytosine to form 5-methylcytosine. DNA methylation is catalyzed by a family of DNA methyltransferases (DNMTs). DNA demethylation by DNA hydroxylase TET enzymes is a critical process for reversing this methylation. The article by Celik et al. discusses recent studies showing that the cooperative regulation of these DNA methylation enzymes is critical for hematopoiesis and leukemogenesis [4]. Acetylation, methylation, and phosphorylation of histones change the 


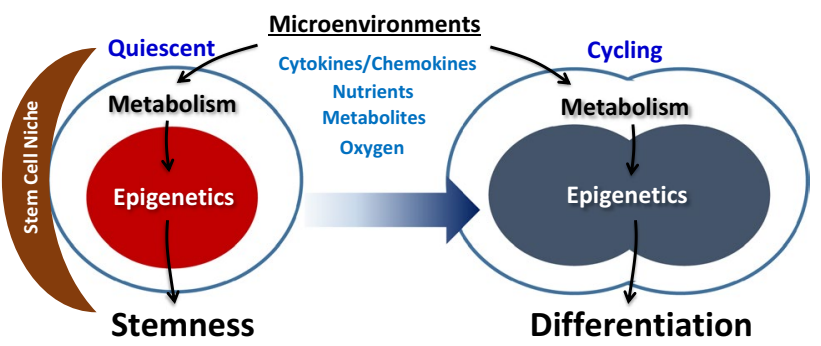

Fig. 1 Multistep processes for control of hematopoiesis. Numerous factors in the microenvironment that affect cell cycle, metabolism, and epigenetics have been identified as regulating HSC homeostasis. Tight connections among these processes are required for normal hematopoiesis, and their dysregulation can cause hematopoietic malignancy

ability of regulatory transcription machinery proteins to access the chromatin of genomic DNA, thereby controlling gene expression. In particular, Polycomb group (PcG) proteins contribute to self-renewal of HSCs by repressing genes involved in differentiation. Whereas dysregulation of such histone modification causes the pathogenesis of myeloid malignancies, PcG proteins also have oncogenic effects in some situations. As described in the review by Takamatsu-Ichihara et al., PcGs have complicated roles in tumorigenesis, but epigenetic regulation mediated by histone modification is critical for hematopoiesis [5].

There are several pathways for crosstalk between metabolism and epigenetics. For example, the $\mathrm{NAD}^{+} / \mathrm{NADH}$ ratio regulates sirtuin histone deacetylases, and acetyl Co-A is used as a donor for HAT-mediated histone acetylation. DNMT and histone methyltransferase add methyl groups from S-adenosyl methionine (SAM) to DNA and histones, respectively. Because SAM is derived from the essential amino acid methionine, the intracellular dynamics of amino acids affect these methylation reactions. In this issue, Inoue et al. discuss an exciting topic in this field: the discovery that gain-of-function mutations of isocitrate dehydrogenases (IDHs) contribute to leukemogenesis [6]. IDH catalyzes the oxidative decarboxylation of isocitrate to $\alpha$-ketoglutarate in the Krebs cycle. The mutant IDH1/2 acquire abnormal activity that converts $\alpha$-ketoglutarate to D-2-hydroxyglutarate (D2HG). This discovery led to the novel concept of "oncometabolites". Interestingly, epigenetic changes are the main targets of the mutated IDHs. D2HG is a competitive inhibitor of $\alpha$-ketoglutarate, and therefore inhibits many dioxygenases, because $\alpha$-ketoglutarate is required for their enzymatic activity. These inhibited dioxygenases include the TET DNA hydroxylases and histone demethylases, which are involved in epigenetic modification. Thus, IDH is a critical molecule linking metabolism and epigenetic status.

This review series highlights the metabolism and epigenetic regulation in hematopoiesis. Deep understanding of the molecular mechanisms of these processes will advance regenerative medicine, anti-aging medicine, and cancer treatments, hopefully in the near future.

\section{References}

1. Nakamura-Ishizu A, Suda T. Aging of the hematopoietic stem cells niche. Int J Hematol. 2014;100:317-25.

2. Yamazaki S, Nakauchi $H$. Bone marrow Schwann cells induce hematopoietic stem cell hibernation. Int $\mathrm{J}$ Hematol. 2014;99:695-8.

3. Jiang Y, Nakada D. Cell intrinsic and extrinsic regulation of leukemia cell metabolism. Int J Hematol. 2016. doi:10.1007/ s12185-016-1958-6.

4. Celik H, Kramer A, Challen GA. DNA methylation in normal and malignant hematopoiesis. Int J Hematol. 2016. doi:10.1007/ s12185-016-1957-7.

5. Inoue $\mathrm{S}$, Lemonnier $\mathrm{F}$, Mak TW. Roles of IDH $1 / 2$ and TET2 mutations in myeloid disorders. Int J Hematol. 2016. doi:10.1007/s12185-016-1973-7.

6. Takamatsu-Ichihara E, Kitabayashi I. The roles of Polycomb group proteins in hematopoietic stem cells and hematological malignancies. Int J Hematol. 2016. doi:10.1007/ s12185-016-2011-5. 